\title{
A Case of Successful Treatment of Septic Shock Secondary to Scrub Typhus
}

\author{
Ahmad Faiq Sa'dan Mohd Zaini and Azlina Ibrahim* \\ Department of Medicine, Hospital Bentong, Pahang, Malaysia
}

\begin{abstract}
Scrub typhus is a tropical disease and endemic problem mostly in rural parts of Malaysia. Typical presentation is acute febrile illness with systemic involvement, Multiple Organ failure and death. The non-specific flu like symptoms, low index of suspicion by clinicians and the unavailability of diagnostic test in most healthcare centres results in under-recognized scrub typhus. We report a case of a traveller to rainforest - National Park, Malaysia who presented with acute febrile illness and septicemic shock with pathognomonic eschar and positive serology test for scrub typhus. The patient recovered after appropriate diagnosis and prompt treatment.
\end{abstract}

Keywords: Scrub Typhus • Eschar • Multiple Organ Failure • Fatal

\section{Introduction}

Scrub typhus is a lethal infectious disease caused by Orientia tsutsugamushi, a gram negative intracellular bacterium which is transmitted to humans through the bite of infected Leptotrombidium mites. Typical presentation is acute febrile illness with non specific symptoms, systemic involvement with severe complications and death [1]. The disease is commonly distributed throughout the Asia Pacific regions including Malaysia $[2,3]$. There were limited data on the prevalence of scrub typhus in Malaysia. However a study on the antibody prevalence Orientia tsutsugamushi to aboriginal population in West Malaysia suggested that scrub typhus remains an important disease amongst various aboriginal subgroups with the highest prevalence being observed for the Pahang subgroup [4,5]. The study concluded environment, socioeconomic and behavioural risk factors have a significant relationship to the risk of exposure to scrub typhus [5]. The study was only included 280 individuals from 7 aboriginal subgroups yet it may not represent the entire native populations [4].

The common clinical manifestations of the disease are fever, headache and myalgia. The diagnosis of scrub typhus can be challenging due to the nature of the disease; non-specific flu like illness which can mimic many other zoonotic diseases. An eschar is a pathognomonic clinical feature of scrub typhus, begins as a papule at the site of chigger feeding and then ulcerates form a black crust like a skin burn from a cigarette [6]. Laboratory methods for diagnosing scrub typhus are mainly based on serological tests and molecular assays with the gold standard test for diagnosis of scrub typhus is the indirect Immunofluorescence Assay (IFA) [6]. The indirect Immunoperoxidase Assay (IIP) serological test is a modification of IFA that provides a comparable sensitivity and specificity in diagnosis of scrub typhus [7]. Scrub typhus can result in severe multiorgan failure failure with a case fatality rate up to $30 \%$ or higher without appropriate treatment [8]. There are limited data on the rate of sepsis in scrub typhus infection however a study done in Northern India emphasized that sepsis with Multiple Organ Dysfunction Syndrome (MODS) is the second most common complications after acute respiratory distress syndrome [9]. We present

*Address for Correspondence: Azlina Ibrahim, Department of Medicine, Hospital Bentong, Pahang, Malaysia, Tel: +609-2223333; E-mail: azlina1984@yahoo.com

Copyright: (C) 2020 Ibrahim A, et al. This is an open-access article distributed under the terms of the Creative Commons Attribution License, which permits unrestricted use, distribution, and reproduction in any medium, provided the original author and source are credited.

Received 02 July 2020; Accepted 16 July 2020; Published 22 July 2020 a case of scrub typhus in a 24-year-old traveller with acute febrile illness and septicemic shock which later found to have pathognomonic eschar and positive serological test for scrub typhus.

\section{Case Report}

A 24-years-old Malaysian man presented to the emergency department with a 9-day history of fever, headache, diarrhea and loss of appetite. He denied cough or runny nose. Two weeks before illness, he travelled to National Park in Pahang, Malaysia for jungle trekking. In the ward, patient was ill looking and lethargic, febrile with temperature of $39^{\circ} \mathrm{C}$, tachycardic with heart rate of 120 beats per minute, blood pressure of $100 / 60 \mathrm{mmHg}$ requiring intravenous noradrenalin $0.5 \mathrm{mcg} / \mathrm{kg} / \mathrm{min}$ with oxygenation saturation of $97 \%$ on room air. Physical examination disclosed presence of two eschar measured $7 \mathrm{~mm}$ below his right axilla and left forearm (Figure 1). Chest auscultation and abdominal examination were unremarkable. Laboratory test revealed no leukopenia, thrombocytopenia or anaemia but elevated serum Aspartate Aminotransferase (AST), Alanine Transferase (ALT) and Lactate Dehydrogenase (Table 1). He was initially given empirical therapy of intravenous ceftriaxone $2 \mathrm{~g}$ daily for possible leptospirosis. However in view of the travelling history to rainforest and presence of eschar, we decided to treat the patient for possible scrub typhus and therefore empirical therapy is switched to tablet doxycycline $100 \mathrm{mg}$ twice daily. Patient showed marked clinical improvement in less than $24 \mathrm{~h}$ of doxycycline where his temperature and blood pressure return to normal and he felt better. He was discharged without complication. During his clinic visit at day- 10 of treatment, his symptoms resolved and the eschars dissapeared. His serology test for scrub typhus antibody lgM test was positive with the titre of 1:100. His leptospirosis serology and blood film for malaria parasite was negative. Blood culture and sensitivity test yielded no growth.
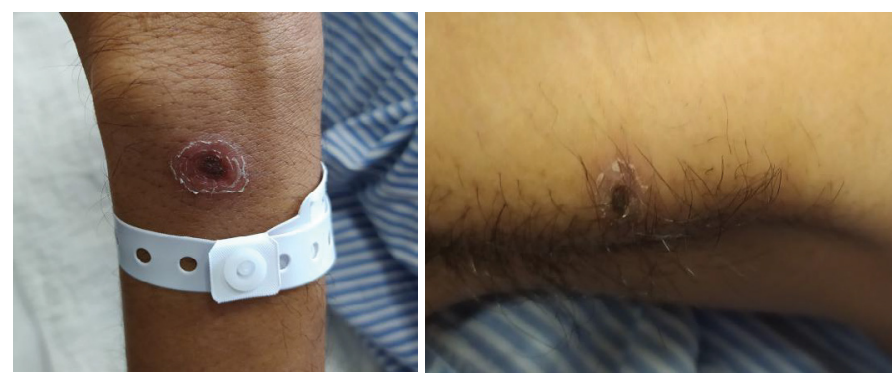

Figure 1. Presence of two eschar measured $7 \mathrm{~mm}$ below his right axilla and left forearm. 
Table 1. Laboratory profile of the patient on admission in Hospital Bentong.

\begin{tabular}{lll}
\hline Variable & Value & Range \\
\hline Hematology & & \\
\hline White blood cell & $9.7 \times 10^{3} / \mathrm{UL}$ & $4.0-11.0$ \\
\hline$\gg \quad$ Neutrophils & $50.20 \%$ & $45.0-75.0$ \\
\hline \multicolumn{1}{c}{ Lymphocytes } & $41.60 \%$ & $24.0-45.0$ \\
\hline Hemoglobin & $14.3 \mathrm{G} / \mathrm{DL}$ & $12.0-18.0$ \\
\hline Platelet & $258 \times 10^{3} / \mathrm{UL}$ & $150-400$ \\
\hline Prothrombin time & $16.9 \mathrm{sec}$ & $14-\mathrm{Dec}$ \\
\hline Activated Partial Thrombin Time (APTT) & 33.7 & $<40$ \\
\hline Blood chemistry & & \\
\hline Alanine Transaminase (ALT) & $159 \mathrm{U} / \mathrm{L}$ & $<45$ \\
\hline Aspartate aminotransferase & $109 \mathrm{U} / \mathrm{L}$ & $<35$ \\
\hline Lactate dehydrogenase & $603 \mathrm{U} / \mathrm{L}$ & $133-225$ \\
\hline Creatinine kinase & $69 \mathrm{U} / \mathrm{L}$ & $<171$ \\
\hline Creatinine & $101 \mathrm{UMOL} / \mathrm{L}$ & $59-104$ \\
\hline Erithrocyte sedimentation rate & $14 \mathrm{MM} / \mathrm{HR}$ & \\
\hline
\end{tabular}

\section{Discussion}

Scrub typhus is a zoonosis caused by Orientia tsutsugamushi, a gramnegative intracellular bacterium. Patient often presented with abrupt onset of fever (normally 8 days to 16 days) following bites from infected mite [10]. Other non specific symptoms include myalgia, rashes and headache which also seen in cases of leptospirosis, dengue, and malaria [9]. In view of the clinical similarities in patients infected with zoonotic disease, the diagnosis of scrub typhus remains a challenge to physicians. Mites are very small and therefore patient may not be aware of the mite bite until they develop eschar. Failure to diagnose scrub typhus results in treatment delay and patient will end up with severe complications such as multiple organ failure, disseminated intravascular coagulopathy and even death.In this case, patient initially given empirical therapy for leptospirosis in view of fever with non specific symptoms for more than a week, history of jungle tracking, swimming, and elevated liver enzyme. However the presence of eschar favours the diagnosis of scrub typhus and patient was promptly manage with doxycycline. Eschar is a valuable clue to the diagnosis of scrub typhus however the overall prevalence of eschar ranged widely in patients infected with the disease [11]. An eschar is not observed in every confirmed cases, therefore history of travelling to or reside in an endemic area is also an important clue to favour the diagnosis of scrub typhus [6]. Clinican must examine patient thoroughly to look for presence of eschar and to get proper travel history especially in areas endemic for scrub typhus [6].

Antibody based serological tests are the mainstay of scrub typhus diagnosis [10]. The older test; Weil-Felix OX-K agglutination reaction test is easy to perform but lack of specificity and sensitivity [12].The Indirect Fluorescent Antibody (IFA) and Indirect Immunoperoxidase (IIP) are the current reference standard and sensitive test for scrub typhus. IFA uses fluorescent anti human antibody to detect specific antibody towards scrub typhus antigen from patient serum whereas the IIP use peroxidase instead of fluorescein for the same manner [13]. Our patient had IIP test for scrub typhus IgM and IgG and the titre was significant for scrub typhus infection. Our patient is treated in Hospital Bentong, which is a district hospital and therefore the IIP test was not available. The lacking of diagnostic test for scrub typhus in district hospital impose a challenge to clinicians. Empirical therapy must be delivered to patient based on clinical judgement and it must be confirmed by the serological test as failure of diagnosis lead to severe complications and death [14].

Acute respiratory distress syndrome is a well known complication for scrub typhus as a result of microangiopathic process leading to focal vasculitis manifest as bronchitis, interstitial pneumonitis or even pleural effusion [14]. Other complications reported include myocariditis, renal failure, elevation of liver enzyme, mucosal hemorrhage and ulcer as well as neurologic complication such as meningitis [14]. A study done in Thailand suggested that septic shock caused by Orientia tsutsugamushi is the most prominent complication in endemic area of scrub typhus. Septic shock results in respiratory failure, disseminated intravascular coagulopathy, renal and hepatic failure [15]. In this case, patient developed septicemic shock with acute transaminitis. Early diagnosis and prompt delivery of the right empirical antimicrobial therapy manage to prevent worse complication.

The mainstay therapy for scrub typhus is tablet doxycycline $200 \mathrm{mg}$ per day. Other treatment options include chloramphenicol, rifampicin and azithromycin [16]. Empirical treatment with doxycycline is justified in endemic area due to the rapid clinical resolution manner following this antibiotic [17]. In cases of doxycycline- resistance strain causing scrub typhus, azithromycin has been advocated to be an effective alternative treatment [18]. In this case, early diagnosis and prompt delivery of correct antimicrobial therapy manage to save the patient and prevent further complications.

\section{Conclusion}

Scrub typhus has appeared as one of the important cause of acute febrile illness which has been neglected for many years [4]. The diagnosis of scrub typhus can be challenging due to the non specific clinical symptoms and signs that can mimic other zoonotic disease. Therefore a thorough history and detailed physical examination are important to aid the diagnosis of scrub typhus. Clinicians must have a high index suspicion of scrub typhus especially to those patients who reside or travel to endemic area. A delay of treatment may result in severe complications and death. A continuous education program and awareness of the disease as one of the differential diagnosis of acute febrile illness in region where the disease are endemic must be advocated.

\section{Declaration of Patient Consent}

The authors certify that they have obtained all appropriate patient consent forms. In the form the patient(s) has/have given his/her/their consent for his/her/their images and other clinical information to be reported in the journal. The patients understand that their names and initials will not be published and due efforts will be made to conceal their identity, but anonymity cannot be guaranteed.

\section{References}

1. Kelly, Daryl, Paul A Fuerst, Wei-Mei Ching and Allen L Richards, et al. "Scrub typhus: the geographic distribution of phenotypic and genotypic variants of orientia tsutsugamushi." Clinical Infectious Diseases 48 (2009): S203-S230.

2. Assad, $F$, Bektimirov $T$, Darwish $R$ and Edlinger $E$, et al. "Rickettsioses: a continuing disease problem." Bulletin of the World Health Organization 60 (1982): 157-164.

3. Watt, George, and Philippe Parola. "Scrub typhus and tropical rickettsioses." Current Opinion in Infectious Disease 16 (2003): 429-436.

4. Tay, ST, S Nazma and M Y Rohani. "Diagnosis of scrub typhus in Malaysian aborigines using nested polymerase chain reaction." The Southeast Asian Journal Tropical Medicine and Public Health 27 (1996): 580-583.

5. Tappe, Dennis, Yvonne Gross, Romano Ngui and Jessica Rauch, et al. "High seroprevalence against typhus group and spotted fever group rickettsiae in rural indigenous populations of peninsular Malaysia." Vector borne and zoonotic diseases 19 (2019): 323-327.

6. Xu, Guang, David Walker, Daniel Jupiter and Peter Melby, et al. "A review of the global epidemiology of scrub typhus." PLoS neglected tropical diseases 11 (2017); (11): e0006062.

7. Scola, La B, and Raoult D. "Laboratory diagnosis of rickettsioses: current approaches to diagnosis of old and new rickettsial diseases." Journal of clinical microbiology 35 (1997); (11): 2715-2727.

8. Ogawa, Motohiko, Toshikatsu Hagiwara, Toshio Kishimoto and Sadashi Shiga 
et al. "Scrub typhus in Japan: epidemiology and clinical features of cases reported in 1998." The American journal of tropical medicine and hygiene 67 (2002): 162-165.

9. Silpapojakul, Kamkarn, Boonyarat Varachit and Khachornsakdi Silpapojakul. "Paediatric scrub typhus in Thailand: a study of 73 confirmed cases." Transactions of the Royal Society of Tropical Medicine and Hygiene 98 (2004): 354-359.

10. Koh, Gavin CKW, Richard J Maude, Daniel H Paris and Paul N Newton, et al. "Diagnosis of scrub typhus." The American journal of tropical medicine and hygiene 82 (2010): 368-370.

11. Kundavaram, AP, Jonathan AJ, Nathaniel SD and Varghese GM, et al. "Eschar in scrub typhus: a valuable clue to the diagnosis." Journal of postgraduate medicine 59 (2013): 177-178.

12. Kelly, DJ, Wong PW, Gan E and Lewis GE, et al. "Comparative evaluation of the indirect immunoperoxidase test for the serodiagnosis of rickettsial disease." The American journal of tropical medicine and hygiene 38 (1988): 400-406.

13. Blacksell, Stuart D, Naomi Bryant J, Daniel Paris $H$ and Jenny Doust A, et al. "Scrub typhus serologic testing with the indirect immunofluorescence method as a diagnostic gold standard: a lack of consensus leads to a lot of confusion." Clinical infectious diseases 44 (2007): 391-401.
14. Rapsang, Amy G, and Prithwis Bhattacharyya. "Scrub typhus." Indian journal of anaesthesia 57 (2013): 127-134.

15. Thap, Lon Chan, Wichai Supanaranond, Sombat Treeprasertsuk and Sirima Kitvatanachai, et al. "Septic shock secondary to scrub typhus: characteristics and complications." The Southeast Asian journal of tropical medicine and public health 33 ('2002); (4): 780-786.

16. Sayed, Iman El, Qin Liu, lan Wee and Paul Hine, et al. "Antibiotics for treating scrub typhus." Cochrane Database of Systematic Reviews 9 (2018); (9): CD002150.

17. Vivekanandan, M, Anna Mani, Yamini Sundara Priya and Ajai Pratap Singh, et al. "Outbreak of scrub typhus in Pondicherry." J Assoc Physicians India 58 (2010): 24-8.

18. Strickman, D, T Sheer, K Salata and J Hershey, et al. "In vitro effectiveness of azithromycin against doxycycline-resistant and -susceptible strains of Rickettsia tsutsugamushi, etiologic agent of scrub typhus." Antimicrob Agents Chemother 39; (1995): 2406-10.

How to cite this article: Ahmad Faiq Sa'dan Mohd Zaini and Azlina Ibrahim. A Case of Successful Treatment of Septic Shock Secondary to Scrub Typhus. Clin Infect Dis 4 (2020) doi: 10.37421/jid.2020.4.119 